\title{
Vestibular And Proprioceptive Alteration Influence Postural Instability During Dual Tasks In Adults Diagnosed With HIV
}

\section{Leah Jamison SPT'1, Gabriel Gines BS, CPT ${ }^{2}$, Jonathan Marshall SPT1 Martin G. Rosario PT, PhD, CSFI, ATRIC Texas Woman's University, Dallas, TX'1. Puerto Rico University, Ponce, PR².}

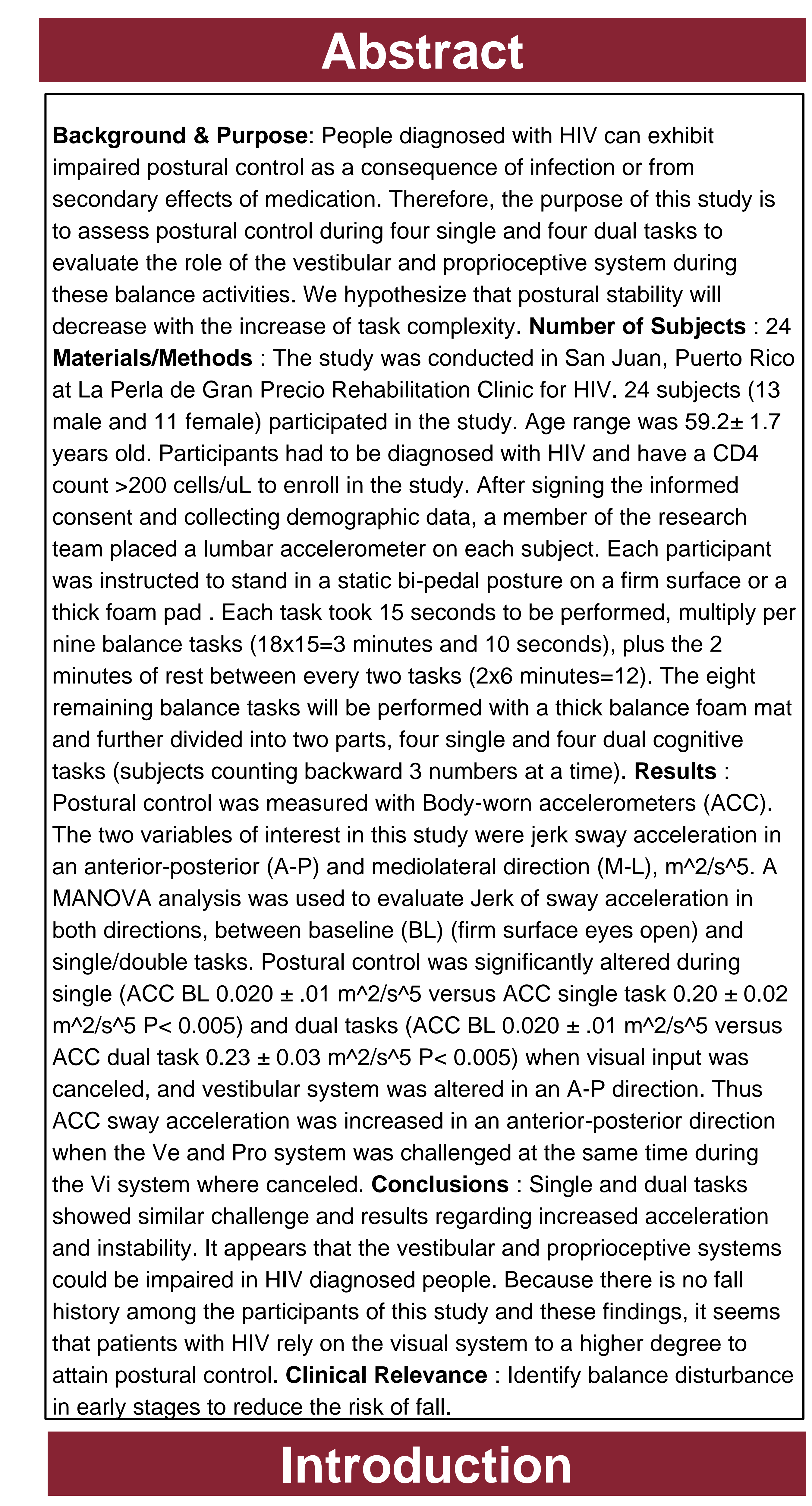

People diagnosed with HIV can exhibit impaired postural control as a consequence of infection or from secondary effects of medication.

How HIV affects the postural control and which sensory system (visual, vestibular somatosensory) is the most affected, possibly vestibular and somatosensory.

Purpose

Assess postural control during single and dual tasks

Characterize the role of the vestibular and proprioceptive system during these balance activities
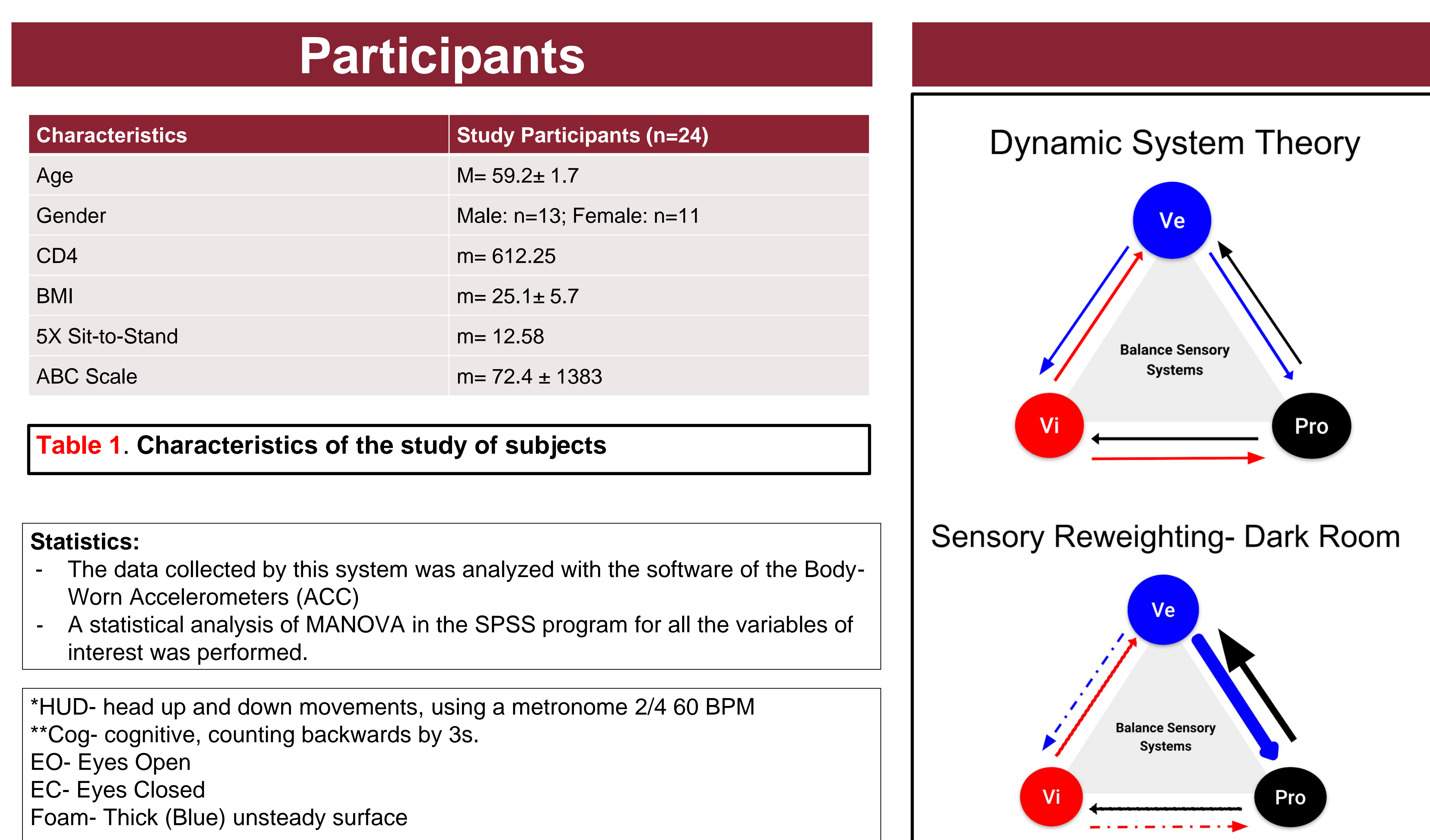

Sensory Reweighting- Dark Room
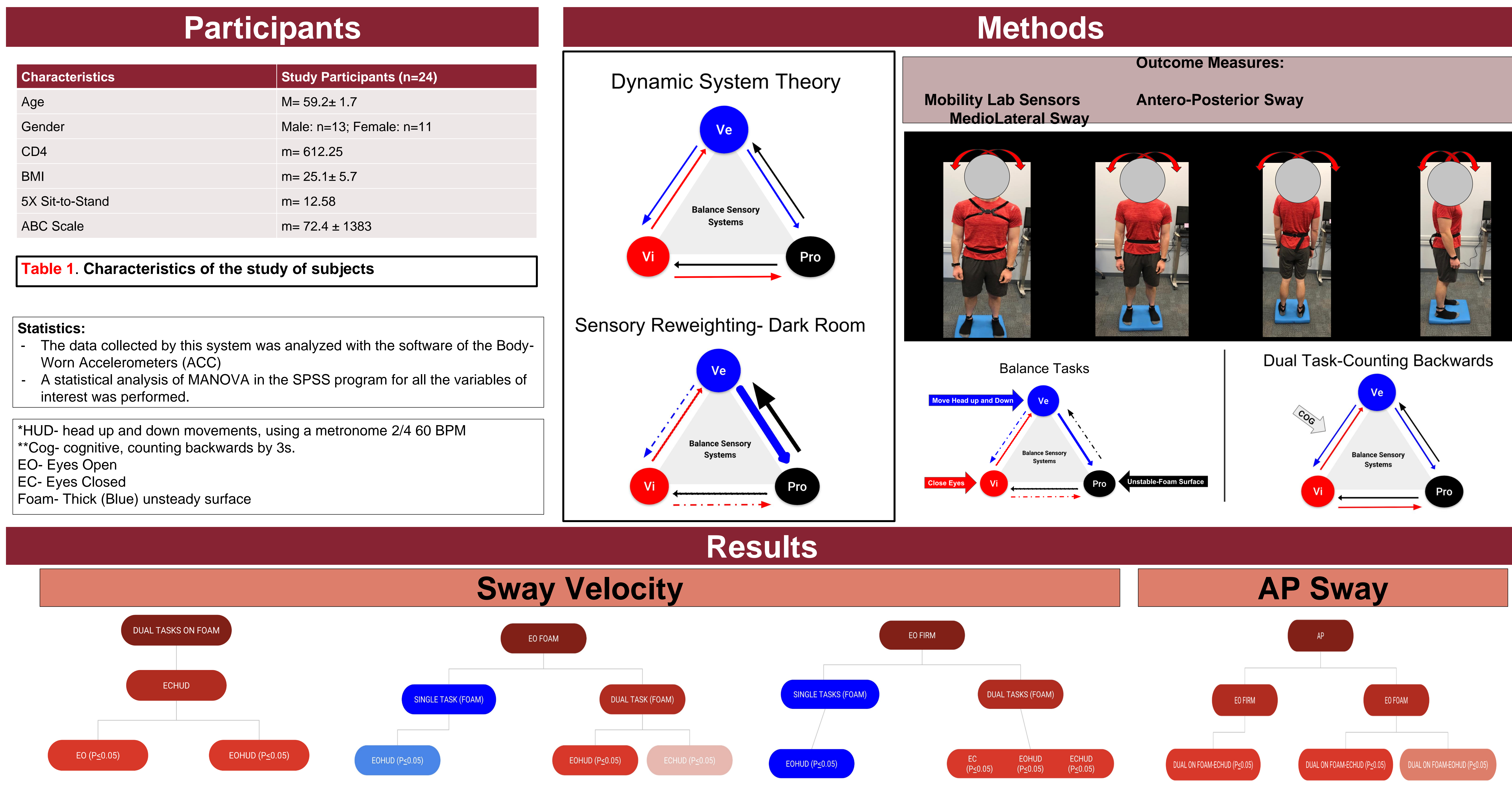

Illustration of ECHUD Dual Cognitive task and
EO + EOHUD comparison

Illustration of EO-FOAM
single tasks comparison

\section{Conclusion}

Dual tasks showed to be a challenge regarding increased acceleration and instability

- It appears that the vestibular and proprioceptive systems could be altered in HIV diagnosed people

- It seems that patients with HIV rely on the visual system to a higher degree to attain postural control before history of falls.

\section{Future Directions}

Dual Task: Sensory-Motor Balance Systems

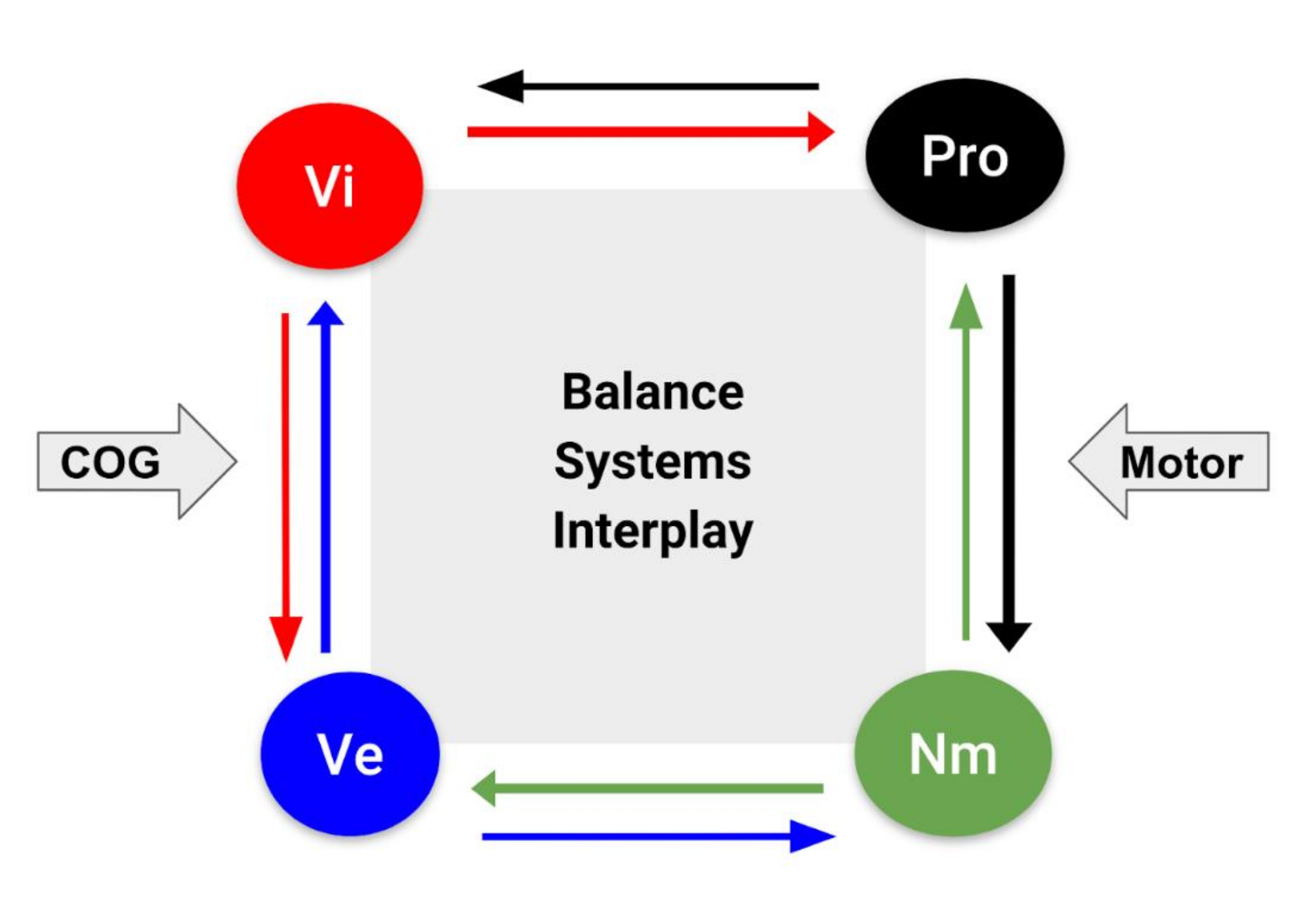

Assess static postural control dual tasks with sensory balance test and lower extremities EMG
Illustration of EO-Firm (left) and EO-Foam (right) single task

\section{Clinical Relevance}

Clinicians should assess balance in early stages to reduce the risk of fall in asymptomatic, normal cd4 levels, undetectable viral load HIV diagnosed individuals.

\section{Reference}

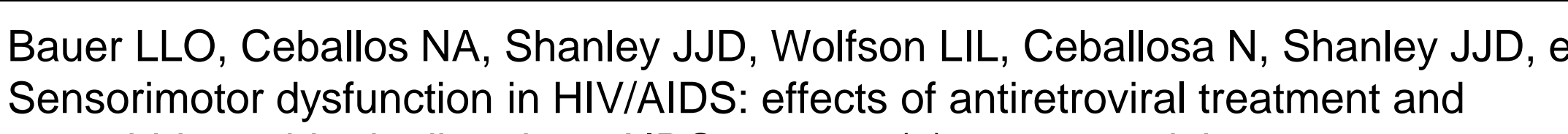

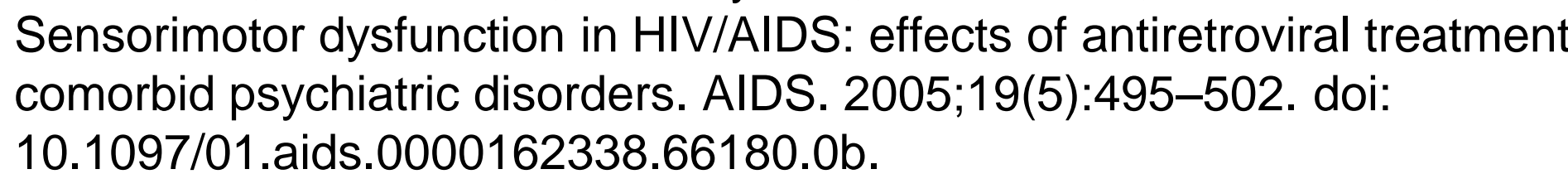

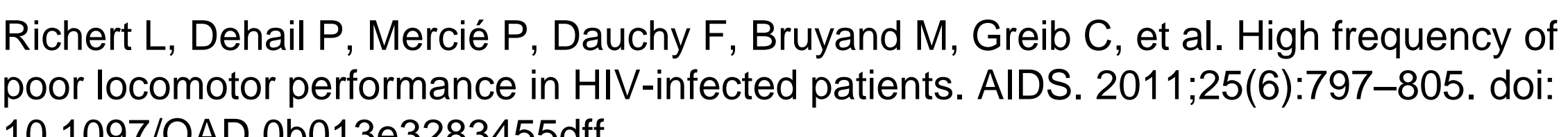

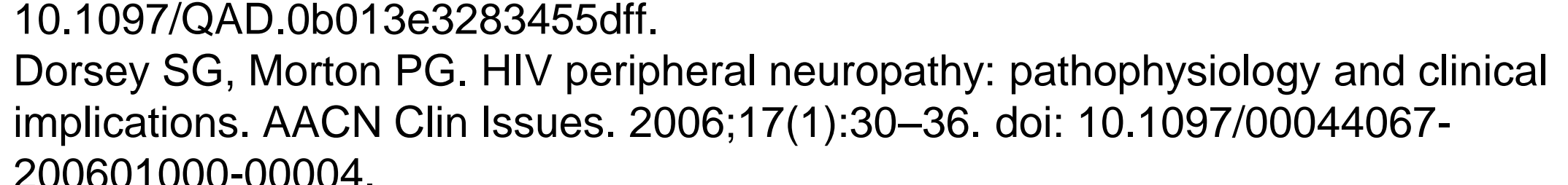

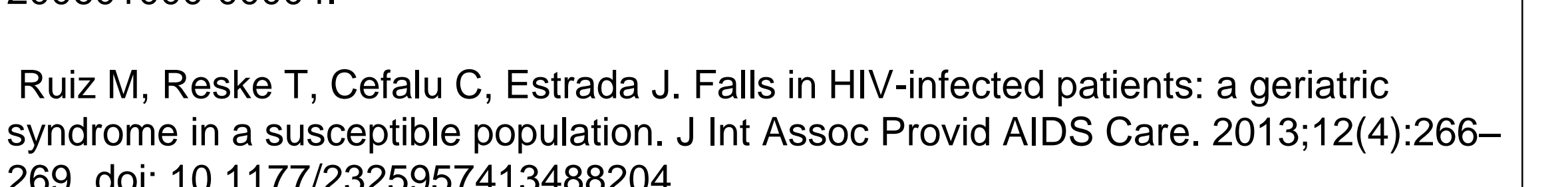

